Annales Geophysicae (2001) 19: 135-145 (C) European Geophysical Society 2001

(5)

\title{
Oxygen abundance in coronal streamers during solar minimum
}

\author{
D. Marocchi ${ }^{1}$, E. Antonucci ${ }^{2}$, and S. Giordano ${ }^{2}$ \\ ${ }^{1}$ Dipartimento di Fisica Generale, University of Torino, Torino, Italy \\ ${ }^{2}$ Osservatorio Astronomico di Torino, Pino Torinese, Italy
}

Received: 1 March 2000 - Revised: 11 September 2000 - Accepted: 7 November 2000

\begin{abstract}
We present a study of the oxygen abundance relative to hydrogen in the equatorial streamer belt of the solar corona during the recent period of activity minimum. The oxygen abundance is derived from the spectroscopic observations of the outer corona performed during 1996 with the Ultraviolet Coronagraph Spectrometer (SOHO) in the ultraviolet region. This study shows that the depletion of oxygen, by almost one order of magnitude with respect to the photospheric values, found in the inner part of streamers by Raymond et al. (1997a) is a common feature of the solar minimum streamer belt, which exhibits an abundance structure with the following characteristics. In the core of streamers the oxygen abundance is $1.3 \times 10^{-4}$ at $1.5 \mathrm{R}_{\odot}$, then it drops to $0.8 \times 10^{-4}$ at $1.7 \mathrm{R}_{\odot}$, value which remains almost constant out to $2.2 \mathrm{R}_{\odot}$. In the lateral bright structures that are observed to surround the core of streamers in the oxygen emission, the oxygen abundance drops monotonically with heliodistance, from $3.5 \times 10^{-4}$ at $1.5 \mathrm{R}_{\odot}$ to $2.2 \times 10^{-4}$ at $2.2 \mathrm{R}_{\odot}$. The oxygen abundance structure found in the streamer belt is consistent with the model of magnetic topology of streamers proposed by Noci et al. (1997). The composition of the plasma contained in streamers is not the same as observed in the slow solar wind. Even in the lateral branches, richer in oxygen, at $2.2 \mathrm{R}_{\odot}$ the abundance drops by a factor 2 with respect to the slow wind plasma observed with Ulysses during the declining phase of the solar cycle. Hence the slow wind does not appear to originate primarily from streamers, with the exception perhaps of the plasma flowing along the heliospheric current sheet.
\end{abstract}

Key words. Interplanetary physics (solar wind plasma) Solar physics, astrophysics and astronomy (corona and transition region; ultraviolet emissions)

\section{Introduction}

The first systematic spectroscopic observations of the streamer belt in the extended corona, above $1.5 \mathrm{R}_{\odot}$, were obtained during solar minimum with the Ultraviolet Corona-

Correspondence to: S. Giordano (giordano@ to.astro.it graph Spectrometer (UVCS) operating on the Solar and Heliospheric Observatory (SOHO). During 1996, the first year of SOHO operations, the streamer belt was very stable and its plane of symmetry was roughly coincident with the solar equatorial plane.

The UVCS observations showed at once a striking difference in the morphology of the quiescent equatorial streamers as observed in the O VI $1032 \AA$ and H I Ly $\alpha 1216 \AA$ emission lines. In the outer corona the structure of the H I Ly $\alpha$ streamers, with maximum brightness on the axis, is roughly consistent with a global dipolar magnetic configuration. The $\mathrm{O}$ VI streamers, on the contrary, have a complex structure consisting of multiple bright regions, according to a quadrupolar or higher order magnetic configuration. The core is dimmed and the elongated bright structures surrounding the dimmed region converge further out at roughly $2.5-3 \mathrm{R}_{\odot}$ to form the interplanetary current sheet.

The most likely interpretation of the dimming of the $\mathrm{O}$ VI emission in the center of streamers is in terms of a local depletion of the oxygen ions (Noci et al., 1997; Raymond et al., 1997a). Raymond et al. have measured the oxygen abundance in a quiescent streamer observed in the outer corona on 23-24 July 1996. Due to the quasi-static, quasi-isothermal nature of this structure the ratio of the intensities of the oxygen and hydrogen emission is proportional to the oxygen abundance relative to hydrogen. The depletion of oxygen in the core of the streamer at $1.5 \mathrm{R}_{\odot}$ is severe. The abundance decreases by an order of magnitude with respect to the photosphere. Also in the streamer branches the oxygen is depleted although to a lesser extent. The relative depletion of oxygen across the streamer is by a factor of 4 (Raymond et al., 1997a). Feldman et al. (1998) and Feldman (1998) found that close to the limb, at $1.03 \mathrm{R}_{\odot}$, the streamer oxygen abundance is close to the photospheric value. Therefore the process that affects this quantity operates further out in the atmosphere. The observed oxygen depletion in streamers agrees with the tendency to find in the corona and in the solar wind an underabundance of heavy elements with high first ionization potential (FIP), $\geq 9 \mathrm{eV}$, relative to elements with lower first ionization potential (Meyer, 1985a,b). The studies of solar composition based on the data obtained more 
recently with the SWICS/Ulysses instrument have revealed however that in the solar wind there is evidence for an overabundance of low-FIP relative to high-FIP elements and the effect is enhanced in the slow solar wind (see Geiss et al., 1995; von Steiger et al., 1995; von Steiger, 1998).

The oxygen depletion in the streamer core has been related to the origin of the slow solar wind by Noci et al. (1997). In this model the slow wind originates in the streamer belt and flows outward along open magnetic field lines which separate the bright $\mathrm{O}$ VI features identified as sub-streamers. The oxygen depletion in this case would originate from the narrowing of the flux tubes before the wind reaches the critical point. A decrease of the flux tube cross-section in the subsonic region reduces the outflow velocity and consequently the flux of protons. Therefore the force dragging the heavy ions in the solar wind, proportional to the proton flux, is also decreasing. When the dynamical friction decreases below the minimum threshold, necessary to carry ions in the solar wind (Geiss et al., 1970), a depletion of oxygen may indeed occur.

On the other hand, because of a certain similarity in the drop of the oxygen abundance in the slow solar wind (Geiss et al., 1995) and in the branches of quiescent streamers, Raymond et al. $(1997 \mathrm{a}, \mathrm{b})$ have proposed the streamer branches as the sources of the slow wind. In this case the severe depletion of oxygen in the center of streamers is explained as an effect of gravitational settling in a closed magnetic field structure. This occurs when the plasma is confined magnetically in the core of a simple one-streamer structure for long periods, of the order of at least a few days. Whereas, in the lateral branches, the plasma would be allowed to flow outward in a time shorter than the time of gravitational settling. In this hypothesis the streamer branches might be channeling some plasma outflow, thus contributing to the slow wind (Raymond et al., 1997b, 1998).

These initial studies have underlined the importance of the oxygen abundance structure within streamers in relation to the understanding of the formation of the slow solar wind. Interpretations by Noci et al. (1997) and by Raymond et al. $(1997 a, b)$ are in support of the overall association of the slow wind with streamers, suggested for instance by Gosling et al. (1981), and would tend to exclude the slow wind forming primarily at the interface between streamers and coronal holes. The aim of the present study is to extend the analysis of the oxygen abundance structure within quiescent streamers to several cases in order to: i) assess whether the difference in the hydrogen-oxygen morphology can be ascribed to an abundance variation as a rule, ii) investigate whether the abundance value is fairly constant in quiescent solar minimum streamers, iii) establish the average abundance structure in the streamer belt in the altitude range from $1.5 \mathrm{R}_{\odot}$ to $2.2 \mathrm{R}_{\odot}$. A further objective is the investigation of the oxygen abundance in relation to the slow wind formation. The analysis is performed considering 5 quiescent streamers observed with the UVCS in the period April-June 1996.

\section{UVCS/SOHO $04 / 11 / 1996$}
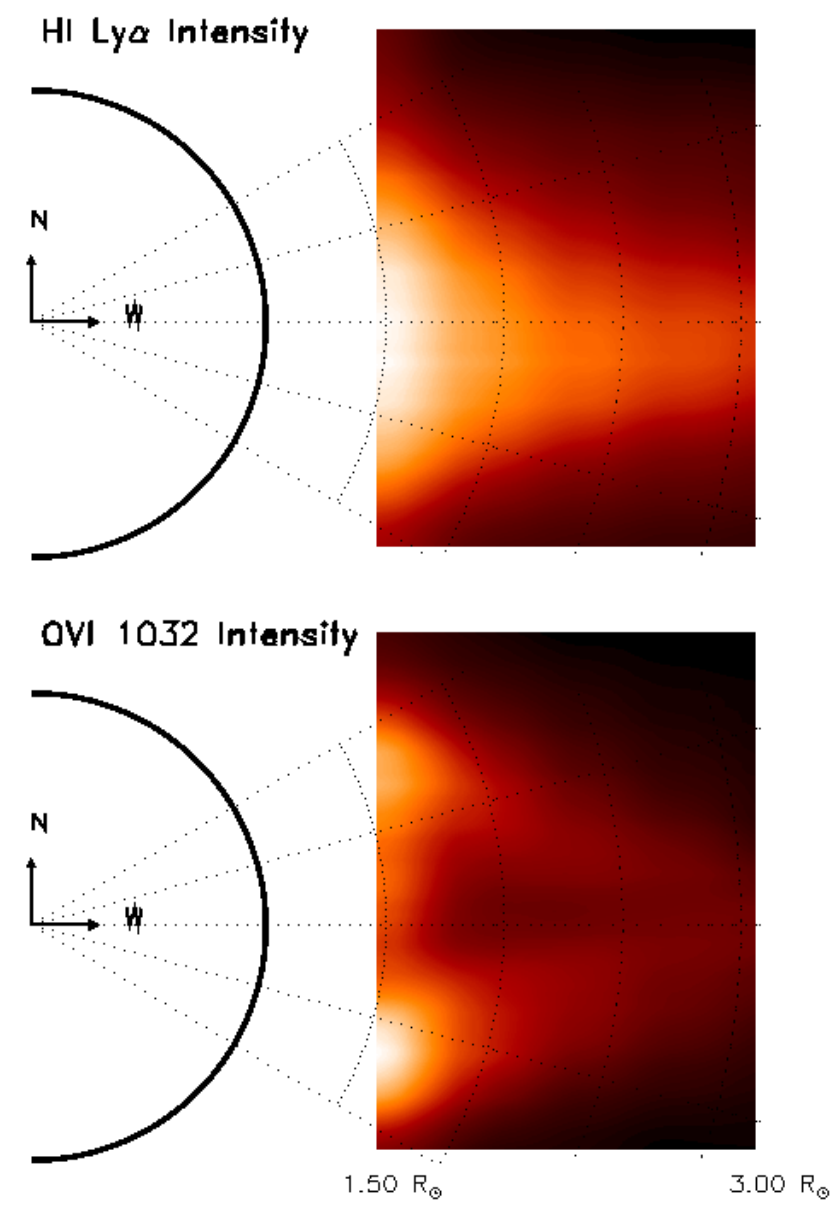

Fig. 1. H I Ly $\alpha$ and O VI 1032 images of the 11 April 1996 equatorial streamer observed between $1.5 \mathrm{R}_{\odot}$ and $3 \mathrm{R}_{\odot}$ with UVCS. The $\mathrm{O}$ VI streamer is certainly formed by two, possibly three, bright structures. The external bright structures are elongated and converge toward the interplanetary current sheet beyond $2.5-3 \mathrm{R}_{\odot}$.

\section{Observations}

We have studied the O VI 1032, $1037 \AA$ and H I Ly $\alpha 1216 \AA$

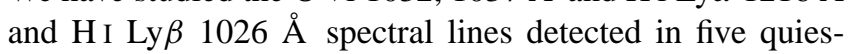
cent equatorial streamers, observed during solar minimum with the UVCS aboard the SOHO spacecraft, in order to determine the oxygen abundance relative to hydrogen in such coronal structures. The UVCS instrument and its capabilities are described by Kohl et al. $(1995,1997)$. The equatorial streamers selected for the analysis were detected on 11, 15 and 18 April 1996 and 2 and 5 June 1996 on the west side of the Sun.

The oxygen and hydrogen spectral lines used in the analysis are detected in the same UVCS channel (the O VI channel), designed to cover the wavelength range from $945 \AA$ to $1123 \AA$ A. This channel also includes a mirror to focus the H I Ly $\alpha$ light on the detector of the O VI channel. The redundant range covers the wavelengths from $1160 \AA$ to $1270 \AA$. 
Table 1. Quiescent streamers used in the analysis

\begin{tabular}{cccc}
\hline Date & Start $(\mathrm{UT})$ & End $(\mathrm{UT})$ & $\mathrm{h}\left(\mathrm{R}_{\odot}\right)$ \\
\hline 11 April 96 & $01: 00: 42$ & $01: 10: 02$ & 1.5 \\
11 April 96 & $01: 10: 56$ & $01: 37: 42$ & 1.7 \\
11 April 96 & $01: 38: 39$ & $01: 51: 28$ & 1.9 \\
11 April 96 & $01: 52: 23$ & $02: 10: 26$ & 2.2 \\
15 April 96 & $23: 18: 16$ & $23: 27: 33$ & 1.5 \\
15 April 96 & $23: 28: 29$ & $23: 55: 17$ & 1.7 \\
15 April 96 & $23: 56: 15$ & $00: 09: 01$ & 1.9 \\
16 April 96 & $00: 09: 57$ & $00: 28: 01$ & 2.2 \\
18 April 96 & $01: 19: 02$ & $01: 45: 49$ & 1.7 \\
02 June 96 & $00: 44: 53$ & $00: 54: 07$ & 1.5 \\
05 June 96 & $01: 53: 54$ & $02: 03: 09$ & 1.5 \\
05 June 96 & $02: 04: 05$ & $02: 30: 53$ & 1.7 \\
05 June 96 & $02: 31: 51$ & $02: 44: 38$ & 1.9 \\
05 June 96 & $02: 45: 33$ & $03: 03: 37$ & 2.2 \\
\hline
\end{tabular}

All lines are observed in the same UVCS channel in order to minimize calibration uncertainties. The streamers were imaged by scanning the corona at variable steps above $1.5 \mathrm{R}_{\odot}$. The UVCS instantaneous field of view (IFOW) is determined by the slit of the spectrometer and the detector mask used in the observation. The IFOW covers an area $(l \times w)$ wide across the radial direction, where $l$ is the portion of the length of the slit selected by the detector mask and $w$ is the width of the slit chosen for the observation. The quantity $l$ is equal to 2500 arcsec for the streamers observed in April and 2350 arcsec for those observed in June 1996, $w$ is equal to 82.5 arcsec, and the position angle of the center of the slit, $\theta_{0}$ (counterclockwise from the North pole), is $270^{\circ}$ in all cases. The date, start and end time of the observation and the heliodistance $h$ of the center of the IFOW are reported in Table I for each observation. Image distortion removal and wavelength and radiometric calibration of the data are performed according to standard procedures (Gardner et al., 1996). The stray-light contribution is negligible in streamers.

The spectral range observed in the oxygen channel has been separated into five spectral intervals, four relative to the spectral lines to be examined plus an additional one chosen to measure the background counting rate. The total intensity, $I$, of each line is obtained in each elementary area along the slit by summing the pixel intensities over the spectral line profile $I=\sum I(\lambda) \Delta \lambda$, after background subtraction. The line intensities can be expressed as functions of the position angle $\theta=\theta_{0}+\Delta \theta$, where $\theta_{0}$ corresponds to the center of the slit, $\Delta \theta$ is defined as $\operatorname{arctg}( \pm y / h)$, and $y$ is the distance from the central position of the slit. As an example, the intensity of the H I Ly $\alpha 1216$ and of the O VI 1032 emission of the streamer observed on 11 April 1996 is imaged in Fig. 1. The analysis of the oxygen abundance is limited to the interval between $1.5 \mathrm{R}_{\odot}$ and $2.2 \mathrm{R}_{\odot}$, where the $\mathrm{HI} \operatorname{Ly} \beta$ line was observed with sufficient statistics.

\section{Oxygen abundance diagnostics}

In the outer corona the O VI 1032, 1037 and H I Ly $\alpha 1216$ and $\operatorname{Ly} \beta 1026$ lines are formed both by collisional excitation and resonant scattering of transition region and chromospheric photons, respectively. The importance of the radiative relative to the collisional contribution increases with heliodistance since the radiative component varies as the density, whereas the collisional contribution decreases as density squared. In the case of the $\mathrm{HI}$ Ly $\alpha 1216$ line the resonant emission is by far the predominant mechanism of line formation in the corona.

In regions where the plasma flows are negligible, the oxygen abundance relative to hydrogen can be derived either from the ratio of the collisional intensities or from the ratio of the radiative components of the O VI 1032 and H I Ly $\beta 1026$ lines (Raymond et al., 1997a):

$$
\begin{aligned}
& \frac{N_{\mathrm{O}}}{N_{\mathrm{H}}} \simeq \frac{I_{\mathrm{OVI}, \text { rad }}}{I_{\mathrm{Ly} \beta, \text { rad }}} \frac{n_{\mathrm{HI}} / n_{\mathrm{H}}}{n_{\mathrm{OVI}} / n_{\mathrm{O}}} \frac{b_{\mathrm{Ly} \beta}}{b_{\mathrm{OVI}}} \frac{f_{\mathrm{Ly} \beta}}{f_{\mathrm{OVI}}} \frac{\delta v_{\mathrm{OVI}}}{\delta v_{\mathrm{Ly} \beta}} \frac{I_{\mathrm{Ly} \beta, \text { disk }}}{I_{\mathrm{OVI}, \mathrm{disk}}}, \\
& \frac{N_{\mathrm{O}}}{N_{\mathrm{H}}} \simeq \frac{I_{\mathrm{OVI}, \text { coll }}}{I_{\mathrm{Ly} \beta, \text { coll }}} \frac{n_{\mathrm{HI}} / n_{\mathrm{H}}}{n_{\mathrm{OVI}} / n_{\mathrm{O}}} \frac{b_{\mathrm{Ly} \beta}}{b_{\mathrm{OVI}}} \frac{q_{\mathrm{Ly} \beta}}{q_{\mathrm{OVI}}},
\end{aligned}
$$

where, $N_{\mathrm{O}} / N_{\mathrm{H}}$ is the abundance of oxygen relative to hydrogen, $I_{\mathrm{O} \text { VI,rad }}, I_{\mathrm{O} \text { VI,coll }}, I_{\mathrm{Ly} \beta, \text { rad }}, I_{\mathrm{Ly} \beta, \text { coll }}$ are the radiative and collisional components of O VI 1032, and H I Ly $\beta 1026$ intensities. The collisional and radiative components of the two lines can be separated with a method which also makes use of the observed intensities of the O VI 1037 and H I Ly $\alpha$ 1216 lines, as discussed in the Appendix. The quantities $n_{\mathrm{HI}} / n_{\mathrm{H}}$ and $n_{\mathrm{OVI}} / n_{\mathrm{O}}$ are the concentrations of $\mathrm{HI}$ atoms and $\mathrm{OVI}$ ions and their values depend on electron temperature; $b_{\mathrm{Ly} \beta}=0.88$ and $b_{\mathrm{OVI}}=1.0$ are the branching ratios for the absorbed photons to be re-emitted in the coronal line; $f_{\mathrm{Ly} \beta}=0.0791$ and $f_{\mathrm{OVI}}=0.131$ are the oscillator strengths; $I_{\mathrm{Ly} \beta, \text { disk }}=4.13 \times 10^{13} \mathrm{ph} \mathrm{cm}^{-2} \mathrm{~s}^{-1} \mathrm{sr}^{-1}$ and $I_{\mathrm{OVI} \text {, disk }}=1.94 \times 10^{13} \mathrm{ph} \mathrm{cm}^{-2} \mathrm{~s}^{-1} \mathrm{sr}^{-1}$ are the disk intensities measured with UVCS, used in the analysis by Raymond et al. (1997a). The ratio $\delta v_{\mathrm{O}} \mathrm{vI} / \delta v_{\mathrm{Ly} \beta}$ of line widths is computed on the assumption that the line broadening is approximately thermal in regions of predominantly closed magnetic field lines. The quantities $q_{\mathrm{Ly} \beta}=6.57 \times 10^{-9} \mathrm{~cm}^{3}$ $\mathrm{s}^{-1}$ and $q_{\mathrm{OVI}}=1.85 \times 10^{-8} \mathrm{~cm}^{3} \mathrm{~s}^{-1}$ are the collisional excitation rates computed at $\log \mathrm{T}=6.2$. The parameters given above all refer to the O VI 1032 line. The atomic parameters are derived according to Landini et al. (1990); Mewe (1972); Mewe and Gronenschild (1981).

The validity of the assumption of negligible plasma flow in quiescent streamers is corroborated by the white light observations performed with LASCO (SOHO) on the coronal density enhancements that are presumably carried by the slow solar wind. Their outflow velocity at about $2 \mathrm{R}_{\odot}$ is still below $50 \mathrm{~km} \mathrm{~s}^{-1}$ (Sheeley et al., 1997). Furthermore, during the solar minimum the outflow velocity, determined in streamers on the basis of the O VI 1037/1032 ratios, does not reach values of the order of $100 \mathrm{~km} \mathrm{~s}^{-1}$ out to 3-4 $\mathrm{R}_{\odot}$ (Kohl et al., 1997; Antonucci et al., 1997). 
In this analysis we have to consider the disk intensities of H I Ly $\alpha$ and H I Ly $\beta$, O VI 1032 and 1037, both to separate the collisional from the radiative contributions to coronal emission (see Appendix) and to derive the oxygen abundance relative to hydrogen but, in the second case, only if the radiative components of $\mathrm{H} \mathrm{I} \mathrm{Ly} \beta$ and O VI 1032 lines are used (Eq. 1). In the observation period considered here the UVCS values used by Raymond et al. (1997a) for the H I Ly $\alpha$ line are consistent with the average value, $\simeq 5.36 \times 10^{15}$ ph $\mathrm{cm}^{-2} \mathrm{~s}^{-1} \mathrm{sr}^{-1}$, obtained with the data of SOLSTICE (SOLar STellar Irradiance Comparison Experiment) on the Upper Atmosphere Research Satellite (Rottman et al., 1993; Woods et al., 1993). The ratios of disk intensities $I_{\mathrm{Ly} \alpha} / I_{\mathrm{Ly} \beta}$, $I_{\mathrm{O} \text { VI } 1032} / I_{\mathrm{O} \text { VI } 1037}, I_{\mathrm{Ly} \beta} / I_{\mathrm{O} \text { VI } 1032}$, measured by Wilhelm et al. (1998) with SUMER/SOHO at solar minimum, differ from the data used in this analysis by less than $2 \%, 5 \%$ and $30 \%$, respectively. Therefore the separation of the radiative and collisional components of the $\mathrm{HI}$ and $\mathrm{O}$ VI lines is negligibly affected by the uncertainties in the disk intensities of the lines. On the other hand, the uncertainty in the absolute value of the coronal oxygen abundance relative to hydrogen is $\leq 30 \%$ but only when derived on the basis of the ratio of the radiative components of $\mathrm{H} \mathrm{I} \mathrm{Ly} \beta$ and O VI 1032 lines.

Formulae 1 and 2 are valid for ionization equilibrium that is a likely condition for the plasma observed in quiescent streamers below $2.2 \mathrm{R}_{\odot}$, since the density is relatively high and flow speeds are either absent or low. The electron temperature is assumed to be $\log T=6.2$ from $1.5 \mathrm{R}_{\odot}$ to $2.2 \mathrm{R}_{\odot}$ (see Raymond et al., 1997a). This is justified by the fact that the ratio $\left(\left(n_{\mathrm{HI}} / n_{\mathrm{H}}\right) /\left(n_{\mathrm{OVI}} / n_{\mathrm{O}}\right)\right)$ does not vary significantly in the range $\log T=6.0-6.2$ that includes typical streamer temperatures (see Gibson et al., 1999). Furthermore above $10^{6} \mathrm{~K}$ the variation of the excitation rates $q$ with temperature is quite small. In particular, in the $\log T=6.0-6.2$ range,

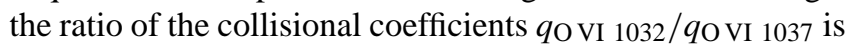
constant; $q_{\mathrm{HI} \mathrm{Ly} \alpha} / q_{\mathrm{HI} \mathrm{Ly} \beta}$ varies no more than $1 \%$ and $q_{\mathrm{O}} \mathrm{VI}$ $1032 / q_{\mathrm{HI} \mathrm{Ly}} \beta$ no more than $8 \%$. The ratios $q_{\mathrm{O} \text { VI } 1032} / q_{\mathrm{O}}$ VI 1037 and $q_{\mathrm{HI} \mathrm{Ly} \alpha} / q_{\mathrm{HI} \mathrm{Ly} \beta}$ are used to separate the collisional and radiative line components (see the Appendix).

The oxygen abundance relative to hydrogen for a quiescent streamer is then directly proportional to the ratio of the $\mathrm{O}$ VI to $\mathrm{H}$ I intensity through a constant factor containing only atomic parameters which weakly depends on the local electron temperature. The abundance is determined independent of electron density.

\section{Oxygen abundance analysis results}

According to the diagnostic methods illustrated in the previous section, for a static plasma at a given temperature the oxygen abundance relative to hydrogen, $N_{\mathrm{O}} / N_{\mathrm{H}}$, is a linear function of the ratios of the collisional/radiative line components, $I_{\mathrm{O} \text { VI,coll }} / I_{\mathrm{Ly} \beta \text {, coll }}$ and $I_{\mathrm{O} \text { VI,rad }} / I_{\mathrm{Ly} \beta \text {,rad. }}$ Therefore, in general, abundance variations are to be expected when the $\mathrm{O}$ VI emission varies relative to the $\mathrm{H}$ I $\mathrm{Ly} \beta$ emission. An example of this correlation is given in Fig. 2, where the oxygen
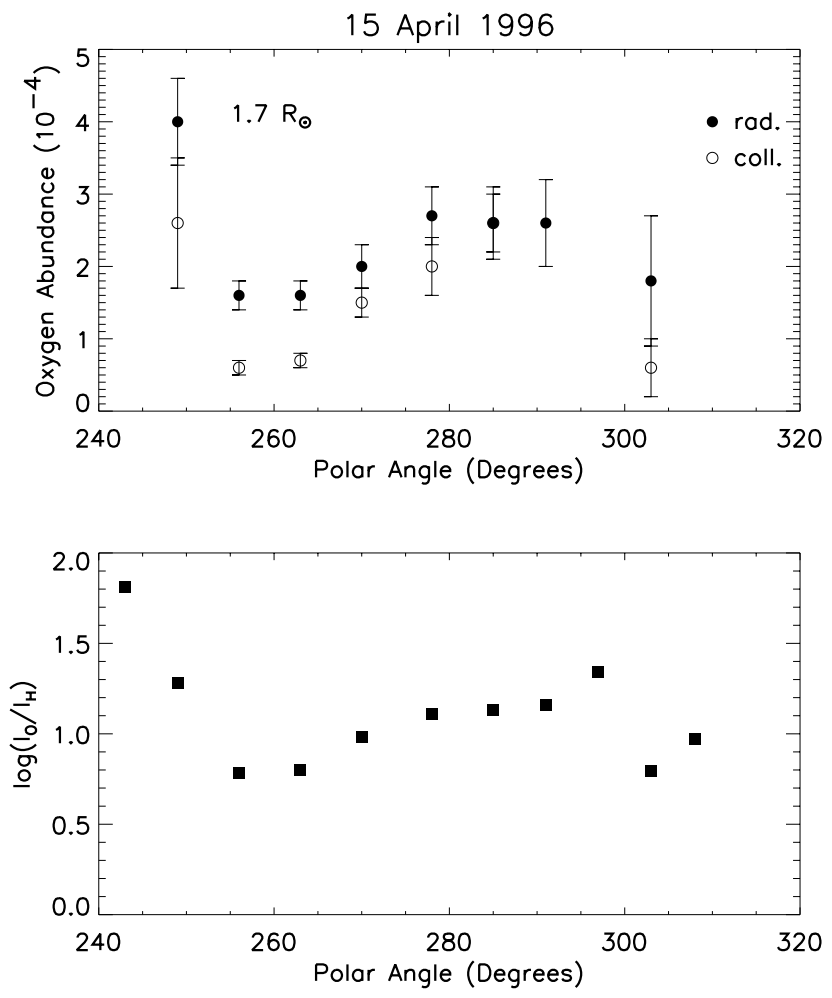

Fig. 2. Top panel: oxygen abundance relative to hydrogen determined at $1.7 \mathrm{R}_{\odot}$ approximately across the axis of the equatorial coronal streamer observed at the West limb on 15 April 1996 (polar angle $270^{\circ}$ corresponds to the equator at the West limb). Bottom panel: relative intensity of the O VI 1032 to the $\mathrm{H} \mathrm{I} \mathrm{Ly} \beta$ line measured at $1.7 \mathrm{R}_{\odot}$ on 15 April 1996 .

abundance variation across the axis of the streamer observed at $1.7 \mathrm{R}_{\odot}$ on 15 April 1996 is described. The instantaneous field of view is divided in 11 equal sectors, 210 arcsec wide. The uncertainty on the intensity $\mathrm{H}_{\mathrm{I}} \operatorname{Ly} \beta$, the weakest line, has a predominant influence on the statistical error of the oxygen abundance.

The results obtained in Fig. 2 indicate that some of the differences between the abundances obtained with the collisional and radiative components are much lower than the maximum uncertainty in the radiative abundance that might be expected from the uncertainty in the ratio of the $\mathrm{H} \mathrm{I} \mathrm{Ly} \beta$ and O VI 1032 disk intensities. This fact implies that possible systematic errors due to disk intensity uncertainties are much lower than $30 \%$.

For each of the five streamers selected for the analysis, the oxygen and hydrogen line intensities are compared to identify regions of maxima and minima of the emission ratio of the two lines (Fig. 3, right panels). The line intensities are then integrated over these regions, or sectors, of the instantaneous field of view in order to decrease the statistical uncertainty when deriving the average oxygen abundance. The streamer boundary is defined as the site where the oxygen intensity drops roughly by $1 / e$. The analysis is performed in the sectors within the streamer where the intensity of the 

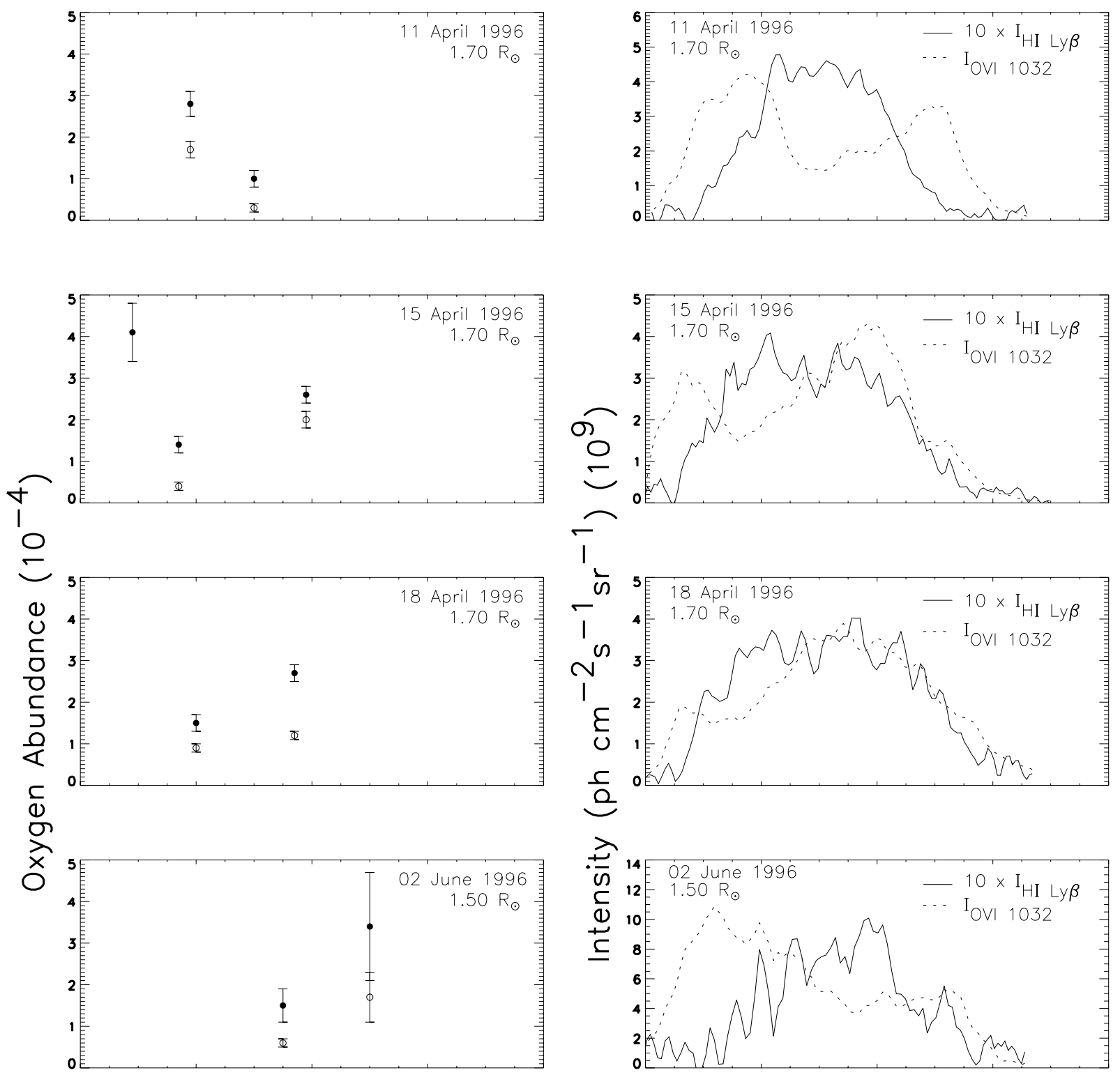

ז
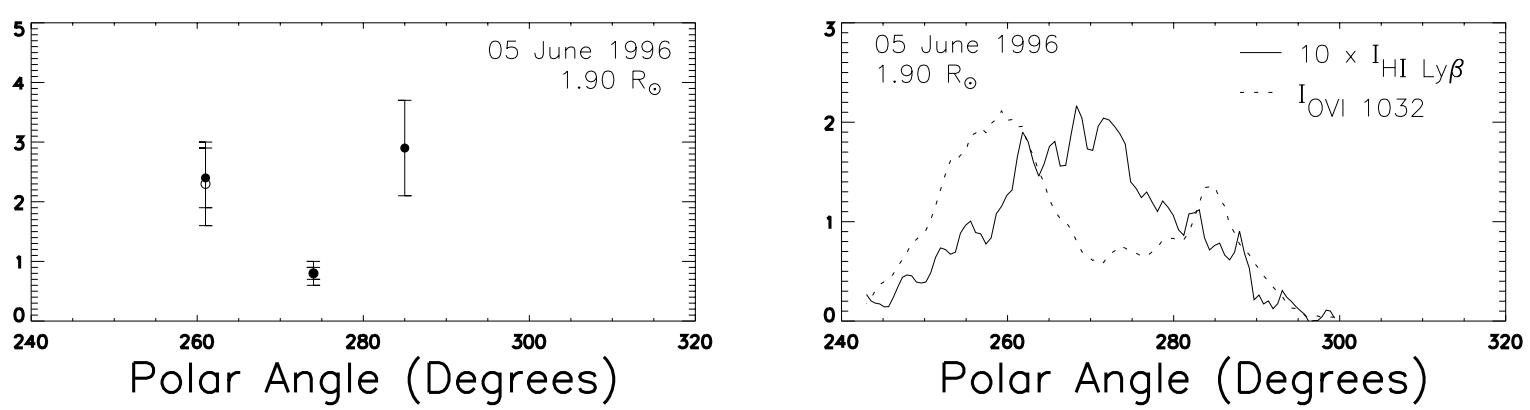

Fig. 3. In the left panels the oxygen abundance relative to hydrogen (averaged over the polar angle sectors reported in Table II) are plotted as full dots and open circles when derived from the radiative and collisional components of the O VI 1032 and the H I Ly $\beta$ lines, respectively. In the right panels the intensity of the $\mathrm{O}$ VI 1032 and the $\mathrm{H} \mathrm{I} \mathrm{Ly} \beta$ lines are compared to show the regions of maximum and minimum $\mathrm{O} / \mathrm{H}$ relative intensity that have allowed the selection of sectors used to perform the abundance analysis. 
H I Ly $\beta$ is sufficiently high.

The abundance results obtained from the analysis of the radiative and collisional emission components are plotted in Fig. 3 (left panels). The heliocentric distance of the instantaneous field of view, the central position, $\theta_{c}$, and width of the selected sectors, the average $I_{\mathrm{Ly} \beta}$ and $I_{\mathrm{O} \text { VI }} 1032$ line intensities, and the average oxygen abundance,

$A_{\mathrm{O} / \mathrm{H}}=\log \frac{N_{\mathrm{O}}}{N_{\mathrm{H}}}+12$,

derived from the ratios of the radiative and collisional components of the O VI and $\mathrm{HI}$ lines within each sector, are reported in Table 2.

The 11 April and 5 June 1996 streamers are roughly symmetrical with respect to the equator $\left(270^{\circ}\right)$ and in this case the oxygen emission is weaker right in the center of the streamer. The other streamers are less symmetrical, probably because their symmetry plane is slightly inclined with respect to the equator and line of sight effects play a role in shaping the coronal structure as observed from SOHO. In all streamers, however, we can identify two lateral branches; bright in O VI although not always developed to the same extent and an inner core region, not necessarily central, that shows a severe oxygen depletion.

\subsection{April 1996 streamer}

The streamer observed on 11 April 1996 (Fig. 1) is an excellent example of the morphological difference between the $\mathrm{HI}$ and $\mathrm{OVI}$ emission in solar minimum conditions. The H I Ly $\alpha$ intensity peaks at the equator, where the O VI 1032 emission drops, by a factor of 3-4 at $1.7 \mathrm{R}_{\odot}$ with respect to the lateral branches (Fig. 3). The $\mathrm{O} / \mathrm{H}$ intensity ratio therefore varies significantly with position angle, with a minimum at the equator and two peaks almost symmetric around the equator. The $\mathrm{O} / \mathrm{H}$ abundance derived for the absolute maximum and central minimum in O VI intensity, from the collisional and radiative $\mathrm{O} / \mathrm{H}$ line ratios, is presented in the same figure. The minima of the $\mathrm{O} / \mathrm{H}$ intensity and abundance are found in the core of the streamer. The abundance relative to the second peak of $\mathrm{O} / \mathrm{H}$ intensity ratio, north of the equator, is not statistically significant. This streamer is well observed out to $2.2 \mathrm{R}_{\odot}$.

\section{$4.2 \quad 15-16$ April 1996 streamer}

The intensity of the $\mathrm{O}$ VI 1032 line measured across the axis of the 15 April 1996 streamer, at $1.7 \mathrm{R}_{\odot}$, shows the same bifurcation observed on 11 April 1996, although not symmetric with respect to the equator. The bifurcation is, as expected, not present in the H I Ly $\beta$ intensity (Fig. 3). The O VI minimum is located about 15 degrees south of the equator. The abundance, derived as an average in the intervals around the minima and the maxima of the $\mathrm{O} / \mathrm{H}$ intensity ratio, confirms that the $\mathrm{O} / \mathrm{H}$ intensity maxima, around $280^{\circ}$ and $245^{\circ}$, are characterized by higher oxygen abundance whereas the region in between is depleted of oxygen. The streamer is an- alyzed out to $2.2 \mathrm{R}_{\odot}$ (the last scan was performed on April 16).

\subsection{April 1996 streamer}

The streamer observed on 18 April 1996 at a distance of $1.7 \mathrm{R}_{\odot}$ from the Sun, shows a dip in the $\mathrm{O} / \mathrm{H}$ intensity ratio displaced $\left(15^{\circ} \mathrm{S}\right)$ from the center of the $\mathrm{H}$ I structure and the southern lateral branch of the streamer is almost absent (Fig. 3). If, however, two sectors, respectively 525 and 273 arcsec wide, the first positioned at $260^{\circ}$ and the second north of the equator close to around $277^{\circ}$, are selected, we still find a decrease of abundance in relation to the drop of $\mathrm{O}$ VI intensity relative to hydrogen emission. The O VI dimming is significant only at $1.7 \mathrm{R}_{\odot}$.

\subsection{June 1996 streamer}

The equatorial streamer of 2 June 1996, whose $\operatorname{H~I~Ly~} \beta$ and O VI 1032 intensities at $1.5 \mathrm{R}_{\odot}$ are shown in Fig. 3, is divided into two sectors, 273 and 315 arcsec wide, both north of the equator and centered on position angle $290^{\circ}$ and $275^{\circ}$, respectively. The minimum of $\mathrm{O} / \mathrm{H}$ intensity ratio at $250^{\circ}$ is neglected since the statistical error in this case is too large due to the very weak intensity of the $\mathrm{H} \mathrm{I} \mathrm{Ly} \beta$ line. The lowest oxygen abundance is again found in the central part of the streamer although not exactly at the equator.

\subsection{June 1996 streamer}

The 5 June 1996 streamer has been studied at four different heights: $1.5,1.7,1.9$ and $2.2 \mathrm{R}_{\odot}$. The correspondence between the maxima of oxygen emission and abundance is also confirmed in this case. The O VI dimming is found right at the equator. The results obtained at $1.9 \mathrm{R}_{\odot}$ are shown in Fig. 3.

\subsection{Average abundance values}

In the core of streamers the oxygen abundance varies between $1.4 \times 10^{-4}$ and $0.6 \times 10^{-4}$ (Fig. 4). These values are obtained by averaging over the regions that present a minimum in the oxygen to hydrogen line intensity ratio. The abundance derived for the lateral branches is plotted versus height in Fig. 5. In these structures the oxygen abundance is larger than in the core and it shows a radial dependence with a value varying from $3.9 \times 10^{-4}$ to $2.1 \times 10^{-4}$ in the range from $1.5 \mathrm{R}_{\odot}$ to $2.2 \mathrm{R}_{\odot}$ (Fig. 5). The streamers which contribute most to the dependence of abundance on heliodistance are the 11 April and 5 June 1996 (Table 1). Since these are the two most symmetric streamers with respect to the equatorial plane, with the maximum of hydrogen intensity at $270^{\circ}$ and symmetric bright lateral branches in O VI, the contributions coming from the lateral branches and the core of streamers are not overlapping when integrating the coronal emission along the line-of-sight. The fact that Fig. 5 mostly reflects the radial variation of streamers symmetric with respect to the equator is confirmed by Fig. 6, where the abundance is 


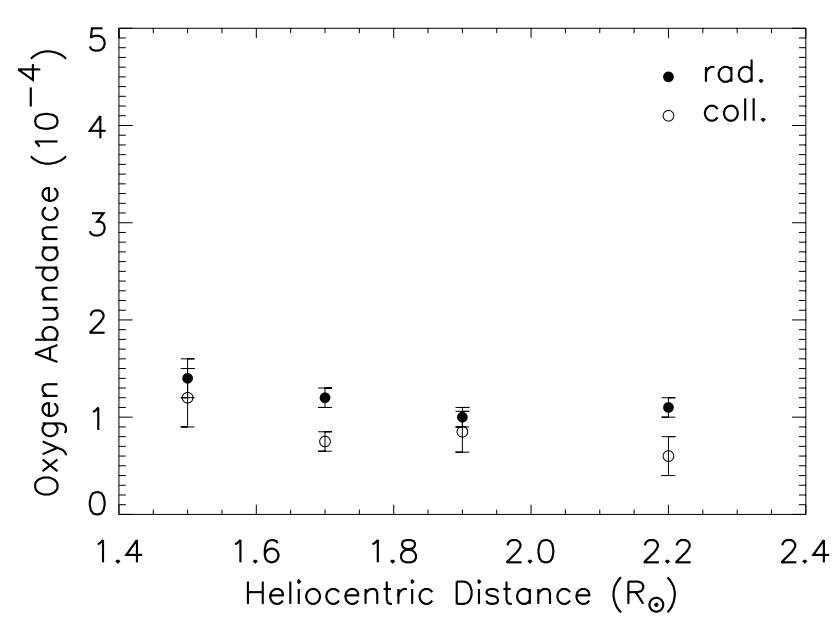

Fig. 4. Average oxygen abundance relative to hydrogen as a function of heliodistance in the streamer core. Full dots and open circles indicate the values derived from the radiative and collisional components of the O VI 1032 and the H I Ly $\beta$ lines, respectively.

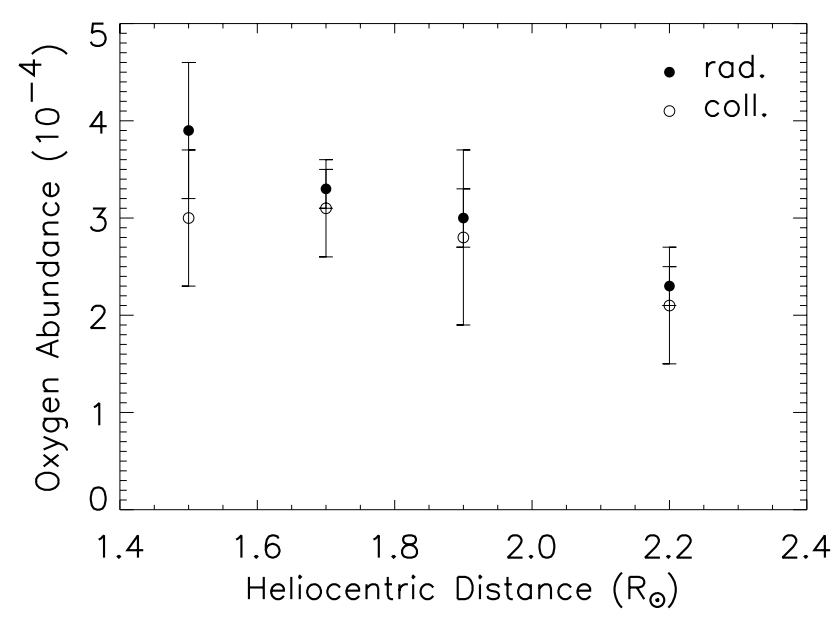

Fig. 5. Average oxygen abundance relative to hydrogen as a function of heliodistance in the streamer lateral structures. Full dots and open circles indicate the values derived from the radiative and collisional components of the O VI 1032 and the H I Ly $\beta$ lines, respectively.

averaged only on the streamers of 11 April and 5 June 1996. The results are obtained by averaging the values derived by the two different methods. Therefore, we can conclude that the dependence of the oxygen abundance on heliodistance found in the lateral branches is real. In these structures, oxygen becomes more rarefied with increasing altitude.

The abundance values obtained from the collisional and radiative line components are plotted separately in Figs. 4 and 5. They only differ significantly in the streamer core at $1.7 \mathrm{R}_{\odot}$ and $2.2 \mathrm{R}_{\odot}$ (Fig. 4). This could be due to uncertainties in the disk intensity of the lines used in the analysis (see Sect. 4). However, since the collisional components weight denser regions, a difference in the abundance derived by means of the two methods might also reveal an

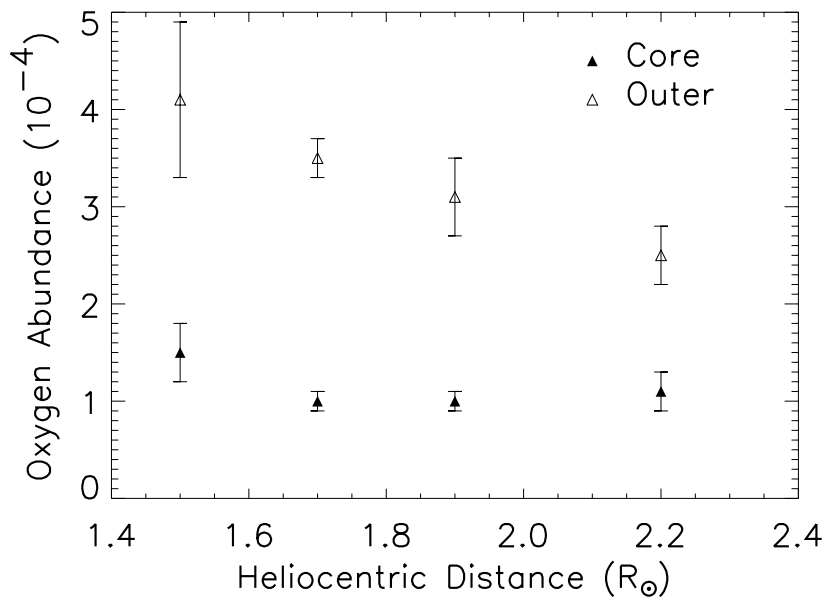

Fig. 6. Oxygen abundance relative to hydrogen as a function of heliodistance obtained by averaging the results from the 11 April and 5 June 1996 data. The streamer core and lateral structures are denoted with full and open triangles, respectively. The values are averages of the results obtained with the ratio of the radiative and collisional components.

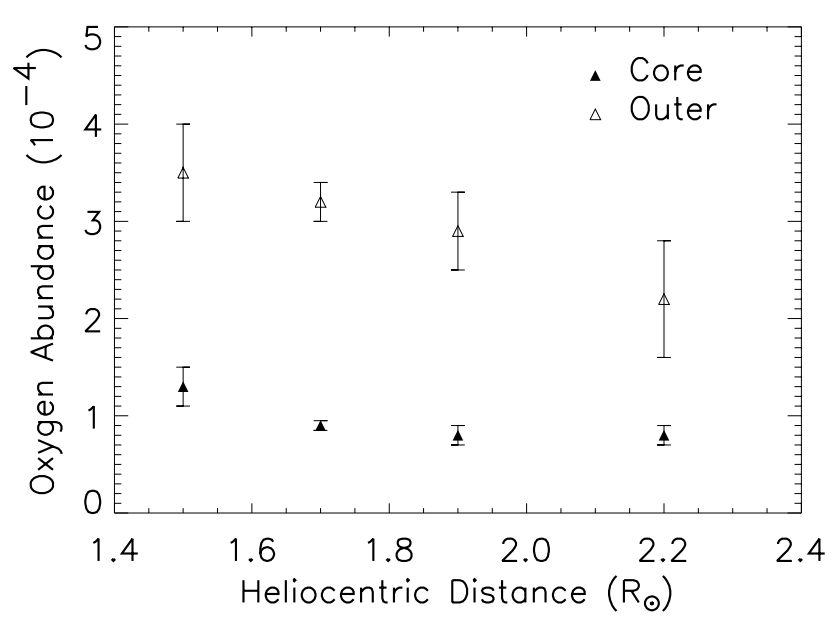

Fig. 7. Oxygen abundance relative to hydrogen as a function of heliodistance in the streamer core and lateral structures, denoted with full and open triangles, respectively. The values are averages of the results obtained with the ratio of the radiative and collisional components.

anti-correlation between electron density and oxygen abundance (Raymond et al., 1997a). Therefore, in the inner part of streamers there might be denser regions characterized by a slightly larger oxygen depletion.

Finally we obtain the characteristic values of the oxygen abundances for core and lateral branches of streamers observed during solar minimum, by averaging the results derived with the two different methods (Fig. 7). 
Table 2. H I Ly $\alpha$ and O VI 1032 Intensities and Oxygen Abundance in Selected Portions of Solar Minimum Quiescent Streamers

\begin{tabular}{|c|c|c|c|c|c|c|c|}
\hline Date & $\begin{array}{c}\text { Heliocentric } \\
\text { distance }\end{array}$ & $\theta_{c}$ sector & $\begin{array}{l}\text { sector } \\
\text { width }\end{array}$ & $\begin{array}{c}I_{\mathrm{Ly} \beta} \\
\left(\mathrm{ph} \mathrm{cm}^{-2} \mathrm{~s}^{-1} \mathrm{sr}^{-1}\right)\end{array}$ & $\begin{array}{c}I_{\mathrm{O} \text { VI } 1032} \\
\left(\mathrm{ph} \mathrm{cm}^{-2} \mathrm{~s}^{-1} \mathrm{sr}^{-1}\right)\end{array}$ & $\begin{array}{l}\log A_{\mathrm{O} / \mathrm{H}} \\
\text { (radiative) }\end{array}$ & $\begin{array}{c}\log A_{\mathrm{O} / \mathrm{H}} \\
\text { (collisional) }\end{array}$ \\
\hline 11 April & $1.5 \mathrm{R}_{\odot}$ & $267^{\circ}$ & $263^{\circ}-271^{\circ}$ & $1.0510^{9}$ & $5.6810^{9}$ & 8.19 & 7.82 \\
\hline 11 April & $1.5 \mathrm{R} \odot$ & $252^{\circ}$ & $249^{\circ}-255^{\circ}$ & $4.6110^{8}$ & $1.0910^{10}$ & 8.66 & 8.67 \\
\hline 11 April & $1.5 \mathrm{R}_{\odot}$ & $288^{\circ}$ & $286^{\circ}-290^{\circ}$ & $6.2910^{8}$ & $7.7410^{9}$ & 8.60 & 8.06 \\
\hline 11 April & $1.7 \mathrm{R}_{\odot}$ & $270^{\circ}$ & $268^{\circ}-273^{\circ}$ & $4.3510^{8}$ & $1.4710^{9}$ & 8.01 & 7.46 \\
\hline 11 April & $1.7 \mathrm{R}_{\odot}$ & $259^{\circ}$ & $253^{\circ}-264^{\circ}$ & $2.9810^{8}$ & $3.7310^{9}$ & 8.46 & 8.23 \\
\hline 11 April & $1.9 \mathrm{R}_{\odot}$ & $272^{\circ}$ & $269^{\circ}-276^{\circ}$ & $2.2910^{8}$ & $6.7910^{8}$ & 8.03 & \\
\hline 11 April & $1.9 \mathrm{R}_{\odot}$ & $285^{\circ}$ & $281^{\circ}-289^{\circ}$ & $6.5510^{7}$ & $1.4210^{9}$ & 8.61 & 8.64 \\
\hline 11 April & $2.2 \mathrm{R} \odot$ & $278^{\circ}$ & $276^{\circ}-279^{\circ}$ & $5.7610^{7}$ & $6.3210^{8}$ & 8.45 & 7.92 \\
\hline 11 April & $2.2 \mathrm{R}_{\odot}$ & $271^{\circ}$ & $269^{\circ}-273^{\circ}$ & $8.0310^{7}$ & $3.5810^{8}$ & 8.06 & 7.59 \\
\hline 11 April & $2.2 \mathrm{R}_{\odot}$ & $265^{\circ}$ & $264^{\circ}-266^{\circ}$ & $8.3010^{7}$ & $7.1210^{8}$ & 8.36 & 7.76 \\
\hline 15 April & $1.5 \mathrm{R}_{\odot}$ & $252^{\circ}$ & $248^{\circ}-256^{\circ}$ & $6.5010^{8}$ & $5.4210^{9}$ & 8.06 & 8.47 \\
\hline 15 April & $1.7 \mathrm{R}_{\odot}$ & $257^{\circ}$ & $255^{\circ}-260^{\circ}$ & $3.2310^{8}$ & $1.6710^{9}$ & 8.16 & 7.62 \\
\hline 15 April & $1.7 \mathrm{R}_{\odot}$ & $249^{\circ}$ & $246^{\circ}-251^{\circ}$ & $1.3510^{8}$ & $2.8510^{9}$ & 8.62 & \\
\hline 15 April & $1.7 \mathrm{R}_{\odot}$ & $279^{\circ}$ & $272^{\circ}-288^{\circ}$ & $2.9710^{8}$ & $3.7510^{9}$ & 8.42 & 8.31 \\
\hline 15 April & $1.9 \mathrm{R}_{\odot}$ & $277^{\circ}$ & $272^{\circ}-283^{\circ}$ & $1.4210^{8}$ & $1.6910^{9}$ & 8.40 & 8.21 \\
\hline 15 April & $1.9 \mathrm{R} \odot$ & $260^{\circ}$ & $259^{\circ}-261^{\circ}$ & $1.5410^{8}$ & $8.2210^{8}$ & 8.05 & 7.94 \\
\hline 16 April & $2.2 \mathrm{R}_{\odot}$ & $276^{\circ}$ & $271^{\circ}-280^{\circ}$ & $5.9410^{7}$ & $6.5310^{8}$ & 8.28 & 8.48 \\
\hline 16 April & $2.2 \mathrm{R}_{\odot}$ & $263^{\circ}$ & $261^{\circ}-265^{\circ}$ & $6.1210^{7}$ & $3.6610^{8}$ & 8.05 & 8.06 \\
\hline 16 April & $2.2 \mathrm{R} \odot$ & $256^{\circ}$ & $253^{\circ}-259^{\circ}$ & $3.5710^{7}$ & $5.3210^{8}$ & 8.35 & \\
\hline 18 April & $1.7 \mathrm{R}_{\odot}$ & $260^{\circ}$ & $255^{\circ}-265^{\circ}$ & $3.3010^{8}$ & $2.0810^{9}$ & 8.18 & 7.98 \\
\hline 18 April & $1.7 \mathrm{R}_{\odot}$ & $277^{\circ}$ & $269^{\circ}-283^{\circ}$ & $3.3610^{8}$ & $3.4610^{9}$ & 8.43 & 8.09 \\
\hline 2 June & $1.5 \mathrm{R} \odot$ & $290^{\circ}$ & $286^{\circ}-295^{\circ}$ & $3.4510^{8}$ & $4.7710^{9}$ & 8.54 & 8.23 \\
\hline 2 June & $1.5 \mathrm{R}_{\odot}$ & $275^{\circ}$ & $269^{\circ}-282^{\circ}$ & $8.4610^{8}$ & $4.5810^{9}$ & 8.19 & 7.76 \\
\hline 5 June & $1.5 \mathrm{R}_{\odot}$ & $293^{\circ}$ & $289^{\circ}-296^{\circ}$ & $3.3710^{8}$ & $7.4210^{9}$ & 8.61 & 8.66 \\
\hline 5 June & $1.5 \mathrm{R} \odot$ & $282^{\circ}$ & $278^{\circ}-287^{\circ}$ & $7.0610^{8}$ & $3.2910^{9}$ & 8.12 & 7.68 \\
\hline 5 June & $1.5 \mathrm{R}_{\odot}$ & $251^{\circ}$ & $247^{\circ}-255^{\circ}$ & $3.3010^{8}$ & $9.9010^{9}$ & 8.55 & \\
\hline 5 June & $1.7 \mathrm{R}_{\odot}$ & $288^{\circ}$ & $286^{\circ}-290^{\circ}$ & $1.7910^{8}$ & $3.1110^{9}$ & 8.57 & 8.44 \\
\hline 5 June & $1.7 \mathrm{R} \odot$ & $275^{\circ}$ & $269^{\circ}-280^{\circ}$ & $3.6210^{8}$ & $1.4010^{9}$ & 7.96 & 7.75 \\
\hline 5 June & $1.7 \mathrm{R}_{\odot}$ & $255^{\circ}$ & $251^{\circ}-258^{\circ}$ & $1.8310^{8}$ & $4.2810^{9}$ & 8.59 & 8.86 \\
\hline 5 June & $1.9 \mathrm{R} \odot$ & $274^{\circ}$ & $270^{\circ}-278^{\circ}$ & $1.6110^{8}$ & $6.7910^{8}$ & 7.91 & 7.91 \\
\hline 5 June & $1.9 \mathrm{R}_{\odot}$ & $285^{\circ}$ & $283^{\circ}-286^{\circ}$ & $8.1110^{7}$ & $1.2910^{9}$ & 8.47 & 8.51 \\
\hline 5 June & $1.9 \mathrm{R}_{\odot}$ & $261^{\circ}$ & $259^{\circ}-263^{\circ}$ & $1.5710^{8}$ & $1.9310^{9}$ & 8.38 & 8.36 \\
\hline 5 June & $2.2 \mathrm{R} \odot$ & $282^{\circ}$ & $281^{\circ}-284^{\circ}$ & $4.4210^{7}$ & $4.7410^{8}$ & 8.32 & 8.40 \\
\hline 5 June & $2.2 \mathrm{R}_{\odot}$ & $274^{\circ}$ & $270^{\circ}-278^{\circ}$ & $6.3810^{7}$ & $3.0310^{8}$ & 8.05 & 7.48 \\
\hline 5 June & $2.2 \mathrm{R}_{\odot}$ & $261^{\circ}$ & $259^{\circ}-263^{\circ}$ & $4.8810^{7}$ & $7.3110^{8}$ & 8.43 & 8.54 \\
\hline
\end{tabular}

\section{Discussion}

The results obtained in the analysis of a few quiescent streamers observed during solar minimum shows that, in an altitude range from $1.5 \mathrm{R}_{\odot}$ to $2.2 \mathrm{R}_{\odot}$, the oxygen abundance structure within the streamer belt follows a regular pattern. The inner part of the streamers is depleted relative to the photospheric value of oxygen by almost an order of magnitude with respect to the photosphere, in agreement with Raymond et al. (1997a). The oxygen abundance relative to hydrogen in the streamer core is close to $1.0 \times 10^{-4}$ while in the photosphere its value is $6.76 \times 10^{-4}$, that is, $A_{\mathrm{O} / \mathrm{H}}=8.83 \pm 0.06$ (see Grevesse and Sauval, 1998). In the lateral branches of the streamers the abundance decreases monotonically with he- liodistance from $3.5 \times 10^{-4}$ (8.54) to $2.2 \times 10^{-4}$ (8.34), that is, oxygen is depleted by a factor of 2 and 3 at $1.5 \mathrm{R}_{\odot}$ and $2.2 \mathrm{R}_{\odot}$, respectively.

The oxygen abundance of streamers can be compared with the value observed in the slow solar wind in the ecliptic plane by Bame et al. (1975), $5.3\left({ }_{-1.0}^{+1.4}\right) \times 10^{-4}$ (8.7). This value was confirmed by the recent SWICS/Ulysses data $\left(5.3\left({ }_{-1.3}^{+2.4}\right) \times 10^{-4}\right)($ von Steiger et al., 1997) during a period of declining activity, between mid-1992 and mid-1993. In this period the solar wind speed was observed to vary periodically between $400 \mathrm{~km} \mathrm{~s}^{-1}$ and $800 \mathrm{~km} \mathrm{~s}^{-1}$. von Steiger et al. (1995) point out that in the same period of declining activity at the magnetic sector boundaries (detected when a crossing of the current sheet formed by the extension of coro- 
nal streamers in the heliosphere occurs) the oxygen abundance can be lower than $4 \times 10^{-4}$. In the fast wind streams, on the other hand, the oxygen abundance, $6.3\left({ }_{-1.5}^{+2.9}\right) \times 10^{-4}$ (8.8), is close to the photospheric value (Wimmer Schweingruber, 1994; Geiss et al., 1995; von Steiger et al., 1995, 1997). Hence, according to the interplanetary observations, the slow wind material found in the ecliptic plane, or within $30^{\circ}$ from the equator (in the case of the Ulysses measurements), is richer of oxygen than the solar minimum equatorial streamers observed with UVCS. The difference in the abundance of the slow solar wind and the lateral structures of quiescent streamers might be not significant only at $1.5 \mathrm{R}_{\odot}$ if possible systematic errors $(\leq 25 \%)$ are taken into account (see Sect. 4). Since the highest depletion of oxygen is found at the heliospheric current sheet, the composition of this layer is the one that differs less from the streamer composition.

Let us assume that the depletion of oxygen in the core of streamers is due to magnetic confinement of the plasma with negligible contribution to the solar wind, according to the suggestion put forward by Raymond et al. (1997b). In this case the plasma is trapped in the closed magnetic field lines of the core region for periods of several days and therefore gravitational settling can occur with a consequent local drop in the abundance of heavy ions. However, the model of a quiescent one-streamer structure where gravitational settling and diffusion of the oxygen ions operate, described by Raymond et al. (1997b), cannot reproduce the observed streamer abundance structure since the drop of oxygen abundance should be larger in the lateral branches.

In fact Raymond et al. (1997b), in order to reconcile the model prediction and the observations, suggest that in the lateral branches gravitational settling does not operate efficiently since the plasma might be confined there for shorter times than in the core and a leakage of mass might occur. In any case, according to the present results, the plasma forming these regions cannot contribute significantly to the slow solar wind flow since the abundance values found at $2.2 \mathrm{R}_{\odot}$, $2.2 \times 10^{-4}$, are lower by more than a factor of 2 than those found in the interplanetary slow wind. Only the tendency, predicted by this model, to find, higher depletion with increasing heliolatitude in closed-magnetic regions is qualitatively consistent with our results.

In the scenario discussed above, the lateral bright branches are at the interface between closed and open field lines. If, however, the morphology suggested by Noci et al. (1997) is representing the real situation, each of the two lateral bright structures would be a sub-streamer characterized by a closed magnetic structure where the plasma could be confined for relatively long time and sub-streamer would be separated by open field lines along which the plasma can flow. If this is the case, only two of the three substreamers needed to form an interplanetary current sheet would extend outward sufficiently to be clearly observed in the extended corona; then the oxygen abundance found in the lateral branches is an average over the closed field line regions where the mechanism of gravitational settling can operate. The observed heliodistance dependence of the oxygen abundance is then consistent with the gravitational settling model by Raymond et al. (1997b) when independently applied to the two substreamers surrounding the core. In the core region, at the site of open magnetic field lines the drag force due to ionproton collision would be acting and the depletion of oxygen in the core of streamers could be ascribed to the mechanism invoked by Noci et al. (1997). The magnetic topology of the open flux tubes, whose cross-section is variable within the streamer, can in fact produce a flow speed smaller than in the radial case while the density remains the same. The proton flux would then be reduced. Since the ions are dragged into the solar wind mainly by Coulomb collisions, the dragging force, proportional to the proton flux, drops. A decrease in proton flux below a certain threshold can, in principle, determine a decrease in the oxygen abundance (Geiss et al., 1970).

It is interesting to note that the material flowing along the flux tube separating substreamers is mainly contributing to the layer where the current sheet forms, a layer that has a magnetic sector boundary as interplanetary signature. von Steiger et al. (1995) have suggested that the material forming the slow wind near sector boundaries shows little sign of static gravitational stratification and is consistent with dynamical stratification. In fact, in the case of gravitational stratification, the drop in the abundance of a heavy ion such as oxygen relative to that of helium would be larger than observed. Helium is light and less affected by the gravitational effect, whereas its drag factor is the least favourable. If gravitational settling is not important to explain the fractionation in the slow wind at the sector boundary, the low oxygen abundance observed with SWICS/Ulysses in proximity to the heliospheric current sheet could then correspond either to the composition of the plasma flowing along the current sheet itself or to that of the plasma in the flow tubes immediately surrounding the coronal streamers and, further out, the current sheet. In the first case, the reduction of proton flux responsible for the oxygen depletion might be due to the magnetic field topology of the open flux tubes within the coronal streamer as suggested by Noci et al. (1997). In the second case, the slow wind might be dynamically fractionated because of the low proton fluxes associated with large geometrical expansion factors of the tubes surrounding the coronal streamers (von Steiger et al., 1995; von Steiger, 1998).

Therefore, we suggest that, in the cases we have analyzed, a complex magnetic topology of quiescent coronal streamers, formed typically by two dominant sub-streamers beyond 1.5 $\mathrm{R}_{\odot}$, is in better agreement with the streamer abundance structure derived from the UVCS observations. Gravitational settling operates in the closed magnetic field regions surrounding the core of the streamer. In the core itself the open magnetic field lines are channeling a slow flow responsible for the depletion of oxygen. In this case the only signature of streamer material in the interplanetary space would be found close to the heliospheric current sheet and presumably interspersed with the slow wind. The alternative scenario, with a closed magnetic field right in the core of streamers, would be consistent with the observed oxygen abundance structure only if the times of magnetic confinement were different for 
the core of the streamer where the material would be confined longer, and for the lateral branches whose material would mix more easily with the plasma flowing along the streamer borders. In both cases, however, the slow solar wind would not originate primarily from streamers but rather from the layers of open field lines surrounding the streamers, where the plasma is flowing outward channeled in flux tubes with large expansion factors.

\section{Appendix Collisional and radiative line intensity components}

In order to separate the collisional and radiative contributions to O VI 1032 and $\mathrm{HI}$ Ly $\beta$ line intensities, the O VI 1037 and $\mathrm{H}$ I Ly $\alpha$ lines must also be considered. The observed H I line intensities are the sum of the collisional and radiative components

$I_{\mathrm{Ly} \alpha, \text { rad }}+I_{\mathrm{Ly} \alpha, \text { coll }}=I_{\mathrm{Ly} \alpha, \text { obs }}$,

$I_{\mathrm{Ly} \beta, \text { rad }}+I_{\mathrm{Ly} \beta, \text { coll }}=I_{\mathrm{Ly} \beta, \text { obs }}$

where $I_{\mathrm{Ly} \alpha, \text { rad }}, I_{\mathrm{Ly} \alpha, \text { coll }}$ and $I_{\mathrm{Ly} \beta, \text { rad }}, I_{\mathrm{Ly} \beta, \text { coll }}$ are the radiative and collisional components of the $\mathrm{H}_{\mathrm{I}} \mathrm{Ly} \alpha$ and $\mathrm{HI} \operatorname{Ly} \beta$ line intensity, respectively. The ratios, $R_{1}$ and $R_{2}$, of the radiative and collisional components of the two lines

$\frac{I_{\mathrm{Ly} \alpha, \text { rad }}}{I_{\mathrm{Ly} \beta, \text { rad }}}=R_{1}$,
$\frac{I_{\mathrm{Ly} \alpha, \text { coll }}}{I_{\mathrm{Ly} \beta, \text { coll }}}=R_{2}$,

are functions of atomic parameters only

$R_{1}=\frac{b_{\mathrm{Ly} \alpha}}{b_{\mathrm{Ly} \beta}} \frac{\lambda_{\mathrm{Ly} \alpha}}{\lambda_{\mathrm{Ly} \beta}} \frac{f_{\mathrm{Ly} \alpha}}{f_{\mathrm{Ly} \beta}} \frac{I_{\mathrm{Ly} \alpha, \text { disk }}}{I_{\mathrm{Ly} \beta, \text { disk }}}$

$R_{2}=\frac{b_{\mathrm{Ly} \alpha}}{b_{\mathrm{Ly} \beta}} \frac{q_{\mathrm{Ly} \alpha}}{q_{\mathrm{Ly} \beta}}$

where $b_{\mathrm{Ly} \alpha}=1.0$ and $b_{\mathrm{Ly} \beta}=0.88$ are the branching ratios for the absorbed photons to be re-emitted in the same line, $f_{\mathrm{Ly} \alpha}=0.416$ and $f_{\mathrm{Ly} \beta}=0.0791$ are the oscillator strengths, $I_{\mathrm{Ly} \beta, \text { disk }}=4.13 \cdot 10^{13} \mathrm{ph} \mathrm{cm}^{-2} \mathrm{~s}^{-1} \mathrm{sr}^{-1}$ and $I_{\text {Ly } \alpha, \text { disk }}=5.24 \cdot 10^{15} \mathrm{ph} \mathrm{cm}^{-2} \mathrm{~s}^{-1} \mathrm{sr}^{-1}$ are the disk intensities measured with UVCS (Raymond et al., 1997a). The collisional excitation rates, $q_{\mathrm{Ly} \alpha}$ and $q_{\mathrm{Ly} \beta}$ depend on electron temperature. The four equations (3-6) relating the radiative and collisional components of the two $\mathrm{H} \mathrm{I}$ lines are sufficient to determine them. Four analogous equations for $\mathrm{O}$ VI allow us to determine the O VI 1032, 1037 radiative and collisional components.

Acknowledgements. We thank Dr. Adriaan van Ballegooijen and Dr. Ruedi von Steiger for helpful discussions and Dr. Carlo Benna for his support in the preparation of the figures. This study was supported by the Agenzia Spaziale Italiana.

The Editor-in-Chief thanks J. C. Raymond and another referee for their help in evaluating this paper.

\section{References}

Antonucci, E., Giordano, S., Benna, C., Kohl, J. L., Noci, G., Michels, J., and Fineschi, S. Signature of open magnetic field lines in the extended solar corona and of solar wind acceleration, Fifth SOHO Workshop ESA SP-404, 175-182, 1997.

Bame, S. J., Asbridge, J. R., Feldman, W. C., Montgomery, M. D., and Kearny, P. D., Solar wind heavy ion abundances, Solar Phys., 43, 463-473, 1975.

Feldman, U., Schüle, U., Widing, K. G., and Laming, J. M., Coronal composition above the solar equator and the North pole as determined from spectra acquired by the SUMER instrument on SOHO, Astrophys. J., 505, 999-1006, 1998.

Feldman, U., FIP effect in the solar upper atmosphere: spectroscopic results, Space Science Reviews, 85, 227-240, 1998.

Gardner, L. D., Kohl, J. L, Daigneau, P. S., Dennis, E. F., Fineschi, S., Michels, J., Nystrom, G. U., Panasyuk, A., Raymond, J. C., Reisenfeld, D. J., Smith, P. L., Strachan, L., Suleiman, R., Noci, G., Romoli, M., Ciaravella, A., Modigliani, A., Huber, M. C. E., Antonucci, E., Benna, C., Giordano, S., Tondello, G., Nicolosi, P., Naletto, G., Pernechele, C., Spadaro, D., Siegmund, O. H. W., Allegra, A., Carosso, P., and Jhabvala, M., Stray light, radiometric and spectral characterization of UVCS/SOHO: laboratory calibration and flight performance, Proc. SPIE, 2831, 2-24, 1996.

Geiss, J., Hirt, P., and Leutwyler, H., On the acceleration and motion of ions in corona and solar wind, Solar Phys., 12, 459-483, 1970.

Geiss, J., Gloeckler, G., von Steiger, R., Balsiger, H., Fisk, L. A., Galvin, A. B., Ipavich, F. M., Livi, S., McKenzie, J. F., Ogilvie, K. W., and Wilken, B., The southern high-speed stream: results from the SWICS instrument on Ulysses, Science, 268, 10331036, 1995.

Gibson, S. E., Fludra, A, Bagenal, F., Biesecker, D., Del Zanna, G., and Bromage, B., Solar minimum streamer densities and temperatures using Whole Sun Month coordinated data sets, J. Geophys. Res., 104, 9691-9700, 1999.

Grevesse, N. and Sauval, A. J., Standard solar composition, Space Science Reviews, 85, 161-174, 1998.

Gosling, J. L., Asbridge, J. R., Bame, S. J., Feldman, W. C., Borrini, G., and Hansen, R. T., Coronal streamers in the solar wind at 1 AU, J. Geophys. Res., 86, 5438-5448, 1981.

Kohl, J. L., Esser, R., Gardner, L. D., Habbal, S., Daigneau, P. S., Dennis, E. F., Nystrom, G. U., Panasyuk, A., Raymond, J. C., Smith, P. L., Strachan, L., Van Ballegooijen, A. A., Noci, G., Fineschi, S., Romoli, M., Ciaravella, A., Modigliani, A., Huber, M. C. E., Antonucci, E., Benna, C., Giordano, S., Tondello, G., Nicolosi, P., Naletto, G., Pernechele, C., Spadaro, D., Poletto, G., Livi, S., Von Der Luhe, O., Geiss, J., Timothy, J. G., Gloeckler, G., Allegra, A., Basile, G., Brusa, R., Wood, B., Siegmund, O. H. W., and Fowler, W., The Ultraviolet Coronagraph Spectrometer for the Solar and Heliospheric Observatory, Sol. Phys., 162, 313356, 1995.

Kohl, J. L., Noci, G., Antonucci, E., Tondello, G., Huber, M. C. E., Gardner, L. D., Nicolosi, P., Strachan, L., Fineschi, S., Raymond, J. C., Romoli, M., Spadaro, D., Panasyuk, A., Siegmund, O. H. W. , Benna, C., Ciaravella, A., Cranmer, S. R., Giordano, S., Karovska, M., Martin, R., Michels, J., Modigliani, A., Naletto, G., Pernechele, C., Poletto, G., and Smith, P. L., First results from the SOHO Ultraviolet Coronagraph Spectrometer, Sol. Phys., 175, 613-644, 1997.

Landini, M. and Monsignori Fossi, B. C., The X-UV spectrum of thin plasmas, Astron. Astrophys. Suppl. Ser, 82, 229-260, 1990. 
Meyer, J. P., The baseline composition of solar energetic particles, Astrophys. J. Suppl., 57, 151-171, 1985a.

Meyer, J. P., Solar-stellar outer atmospheres and energetic particles, and galactic cosmic rays, Astrophys. J. Suppl., 57, 173-204, 1985b.

Mewe, R., Interpolation formulae for the electron impact excitation of ions in the $\mathrm{H}-, \mathrm{He}-, \mathrm{Li}-$, and $\mathrm{Ne}-$-sequences, Astron. Astrophys, 20, 215-221, 1972.

Mewe, R. and Gronenschild, E. H. B. M., Calculated X-radiation from optically thin plasmas IV. Atomic data and rate coefficients from spectra in the range 1-270 ̊. Astron. Astrophys. Suppl. Ser, 45, 11-52, 1981.

Noci, G., Kohl, J. L., Antonucci, E., Tondello, G., Huber, M. C. E., Fineschi, S., Gardner, L. D., Korendyke, C. M., Nicolosi, P., Romoli, M., Spadaro, D., Maccari, L., Raymond, J. C., Siegmund, O. H. W., Benna, C., Ciaravella, A., Giordano, S., Michels, J., Modigliani, A., Naletto, G., Panasyuk, A., Pernechele, C., Poletto, G., Smith, P. L., and Strachan, L., The quiescent corona and slow solar wind, ESA SP-404, 75-84, 1997.

Raymond, J. C., Kohl, J. L., Noci, G., Antonucci, E., Tondello, G., Huber, M. C. E., Gardner, L. D., Nicolosi, P., Fineschi, S., Romoli, M., Spadaro, D., Siegmund, O. H. W., Benna, C., Ciaravella, A., Cranmer, S., Giordano, S., Karovska, M., Martin, R., Michels, J., Modigliani, A., Naletto, G., Panasyuk, A., Pernechele, C., Poletto, G., Smith, P. L., Suleiman, R. M., and Strachan, L., Composition of the coronal streamers from the SOHO Ultraviolet Coronagraph Spectrometer, Sol. Phys., 175, 645-665, 1997a.

Raymond, J. C., Suleiman, R. M., van Ballegooijen, A., and Kohl, J. L., Absolute abundances in streamers from UVCS, ESA SP-415, 383-386, 1997b.
Raymond, J. C., Suleiman, R. M., Kohl, J. L., and Noci, G., Elemental abundances in coronal structures, Space Science Reviews, 85, 283-289, 1998.

Rottman, G. J., Woods, T. N., and Sparn, T. P., Solar-Stellar Irradiance Comparison Experiment 1: 1. Instrument Design and Operation, J. Geophys. Res., 98, 10667-10677, 1993.

Sheeley, N. R., Wang, Y.-M., Hawley, S. H., Brueckner, G. E., Dere, K. P., Howard, R. A., Koomen, M. J., Korendyke, C. M., Michels, D. J., Paswaters, S. E., Socker, D. G., St. Cyr, O. C., Wang, D., Lamy, P. L., Llebaria, A., Schwenn, R., Simnett, G. M., Plunkett, S., and Biesecker, D. A., Measurements of flow speeds in the corona between 2 and $30 \mathrm{R}_{\odot}$, Astrophys. J. Lett, 484, 472-478, 1997.

Wilhelm, K., Marsch, E., Dwivedi, B. N., Hassler, D. M., Lemaire, P., Gabriel, A. H., and Huber, M. C. E, The solar corona above polar coronal holes as seen by SUMER on SOHO Astrophys. J., 500, 1023-1038, 1998.

Woods, T. N., Rottman, G. J., and Ucker, G. J., Solar-stellar irradiance comparison experiment 1: 2 . Instrument design and operation, J. Geophys. Res., 98, 10679-10694, 1993.

von Steiger, R., Wimmer Schweingruber, R. F., Geiss, J., and Gloeckler, G., Abundance variations in the solar wind, Adv. Space Res., 15, 3-12, 1995.

von Steiger, R., Geiss, J., and Gloeckler, G., Composition of the solar wind, Cosmic Winds and the Heliosphere, University of Arizona Press, 581-616, 1997.

von Steiger, R., Composition aspects of the upper solar atmosphere, Space Science Reviews, 85, 407-418, 1998.

Wimmer Schweingruber, R. F., Oxygen, helium and hydrogen in the solar wind: SWICS/Ulysses results, $\mathrm{Ph}$. D. Thesis, University of Bern, 1994. 